\title{
The Association between High Body Mass Index and Mortality Risk in Hospitalized Patients Diagnosed with COVID-19: A Meta-Analysis
}

\author{
Yuzana Maung1), Eti Poncorini Pamungkasari'), Bhisma Murti') \\ 1)Masters Program in Public Health, Universitas Sebelas Maret \\ 2)Faculty of Medicine, Universitas Sebelas Maret
}

\section{ABSTRACT}

Background: COVID-19, caused by severe acute respiratory coronavirus 2 (SARS-CoV-2), emerged as a global pandemic with confirmed death cases of more than 1.27 million worldwide. Since the past pandemic, several studies discovered the adverse effects of excess fat accumulation on the severity of viral infections. This study aimed to investigate the association between high body mass index (BMI) and mortality risk in hospitalized COVID-19 patients based on four categories of high $\mathrm{BMI}$ ranges.

Subjects and Method: A systematic review and Meta-Analysis was conducted using search articles from electronic databases including PubMed, Google Scholar, and Scopus, published from 2019 to 2021. The keywords used to retrieve articles were "Body Mass Index" OR BMI OR Obesity OR Overweight) AND (Mortality OR Death) AND COVID-19 OR SARS-CoV-2. The included studies were fulltext articles published in the English language, reporting adjusted odds ratios from multivariate analysis. The eligibility criteria were defined using PICO model. The article selection was conducted using PRISMA flow chart. The included studies were analyzed by the Review Manager 5.3 application.

Results: A total of nine cohort studies involving 12,907 COVID-19 patients with high BMI conducted from America, Europe, and Georgia regions was included in qualitative synthesis and Meta-Analysis. The pooled estimate showed hospitalized COVID-19 patients with morbid/ class III obesity had a higher risk of mortality $(\mathrm{aOR}=1.77 ; 95 \% \mathrm{CI}=$ 1.27 to $2.47 ; \mathrm{p}=0.001)$ than overweight $(\mathrm{aOR}=$ $1.10 ; 95 \% \mathrm{CI}=1.00$ to $1.21 ; \mathrm{p}=0.060$ ), class $\mathrm{I}$ $(\mathrm{aOR}=1.16 ; 95 \% \mathrm{CI}=0.87$ to $1.55 ; \mathrm{p}=0.300)$, and class II obesity $(\mathrm{aOR}=1.54 ; 95 \% \mathrm{CI}=1.11$ to 2.13; $\mathrm{p}=0.009$ ).

Conclusion: High BMI increases the risk of mortality in hospitalized COVID-19 patients. A potential dose-response relationship may exist between different categories of high BMI range and mortality risk in COVID-19 patients.

Keywords: BMI, obesity, mortality, COVID-19, SARS-CoV-2

\section{Correspondence:}

Yuzana Maung. Masters Program in Public Health, Universitas Sebelas Maret. Jl. Ir. Sutami 36A, Surakarta 57126, Central Java. Email: yuzmg5699@gmail.com. Mobile: +6281295346614 .

\section{Cite this as:}

Maung Y, Pamungkasari EP, Murti B (2021). The Association between High Body Mass Index and Mortality Risk in Hospitalized Patients Diagnosed with COVID-19: A Meta-Analysis. J Epidemiol Public Health. 06(01): 70-82. https://doi.org/10.26911/jepublichealth.2021.06.01.08.

c) (i) Journal of Epidemiology and Public Healthis licensed under a Creative Commons c. ${ }_{\mathrm{EY}}$ NC SA Attribution-NonCommercial-ShareAlike 4.0 International License.

BACKGROUND
COVID-19, caused by the severe acute
respiratory coronavirus 2 (SARS-CoV-2),
was first discovered in December 2019 in

Wuhan, China (WHO, 2020). Since the first outbreak, the rapid transmission of the disease resulted in over 127 million confirmed cases of COVID-19 worldwide, 
including 2,787,593 deaths in March 2021. The most death caused by COVID-19 occurred in the Americas (1,340,530), followed by Europe $(961,435)$, South-East Asia (218,604), Eastern Mediterranean (157,852), Africa $(77,688)$, and Western Pacific $(31,471)$ (WHO, 2021).

The clinical spectrum of SARS-CoV-2 ranged from no symptoms to severe illness, and many may even die. The most common symptoms were fever, cough, fatigue, shortness of breath, and muscle soreness (Chen et al., 2020). In severe cases, rapid progression to acute respiratory distress syndrome (ARDS), septic shock, blood clotting dysfunction, multiple organ failure, and even death has occurred (Tsai et al., 2021).

Obesity caused by excess fat accumulation is one of the most serious diseases affecting people's health. It can cause morbidity by developing a wide range of non-communicable diseases and increasing the risk of infectious diseases (Wilding and Ralston, 2021). Several studies linked the negative impact of obesity on the severity of viral infections, including MERS, H1N1 influenza, and other seasonal influenza (Wilding and Ralston, 2021; Jain and Chaves, 2011).

Furthermore, in the case of COVID-19 infection, some studies discovered obesity as a risk factor for adverse outcome, including the need for hospitalization, invasive mechanical ventilation, intubation, and death (Docherty et al., 2020; Giacomelli et al., 2020; Hajifathalian et al., 2020; Petrilli et al., 2020). Abnormal fat accumulation is related to increased risk of thromboembolism, cytokine storm, decreased respiratory system functions, and an increased risk of acute organ injuries, leading to fatal outcomes in COVID-19 patients (Mafort et al., 2016; Mehta et al., 2020).
However, despite recent epidemiological studies discussing the impact of obesity on mortality risk in COVID-19 patients, the pooled effect from different BMI categories has not yet been evaluated to the best of the author's knowledge. Therefore, this study aimed to investigate the association between high BMI and mortality risk in hospitalized COVID-19 patients based on four categories of high BMI ranges using systematic review and Meta-Analysis.

\section{SUBJECTS AND METHOD}

\section{Study Design}

This was a systematic review and MetaAnalysis of observational cohort studies comparing the high BMI with normal BMI on the risk of mortality in hospitalized COVID-19 patients. A comprehensive search was conducted to collect the relevant articles from the electronic database such as PubMed, Google Scholar, and Scopus, published from 2019 to 2021. The following keywords were used to retrieve articles: "Body Mass Index" OR BMI OR Obesity OR Overweight) AND (Mortality OR Death) AND COVID-19 OR SARS-CoV-2. Preferred Reporting Items for Systematic Reviews and Meta-analyses (PRISMA) guideline was used reporting this Meta-Analysis.

\section{Inclusion Criteria}

The articles were included with following reasons: (1) Full-text articles with observational cohort study published in the English language; (2) Studies emphasized on mortality risk of COVID-19 patients based on four different categories of high BMI range; and (3) The reported results were from multivariate analysis with adjusted odds ratios (aOR).

\section{Exclusion Criteria}

The articles were excluded with following reasons: (1) Articles reported only crude odds ratio (OR) resulted from bivariate 
analysis; (2) The effect measure used was aRR/ aHR, rather than aOR; (3) Reported outcomes other than mortality related to high BMI in COVID-19 patients.

\section{Operational Definition of Variables}

Mortality was defined by the status of death in hospitalized patients diagnosed with COVID-19. It was categorized as dead or alive. The instrument used was document confirmation of death related to COVID-19.

High Body Mass Index (BMI) means BMI above the normal or healthy weight range of $18.5-24.9\left(\mathrm{~kg} / \mathrm{m}^{2}\right)$. BMI was categorized into overweight $\left(25.0-29.9 \mathrm{~kg} / \mathrm{m}^{2}\right)$, class I obesity $\left(30-34.9 \mathrm{~kg} / \mathrm{m}^{2}\right)$, class II obesity $\left(35-39.9 \mathrm{~kg} / \mathrm{m}^{2}\right)$, and class III/ morbid obesity $\left(\geq 40 \mathrm{~kg} / \mathrm{m}^{2}\right)$. The instrument used was the body weighing scale and stadiometer.

\section{Data Analysis}

The data processing was carried out using the Review Manager (RevMan 5.3). The association between high BMI and mortality risk in COVID-19 patients was evaluated based on BMI categories: overweight, class I obesity, class II obesity, and morbid/ class III obesity. Odds ratios with 95\% CIs were calculated from adjusted ORs. The Forest plot was drawn to present pooled estimate effect of high BMI. The heterogeneity among included studies was presented by the intuitive index $\left(\mathrm{I}^{2}\right)$. Fixed effect model Meta-Analysis was used for homogenous data and random effect model for heterogeneous data across studies. Publication bias was investigated using a funnel plot.
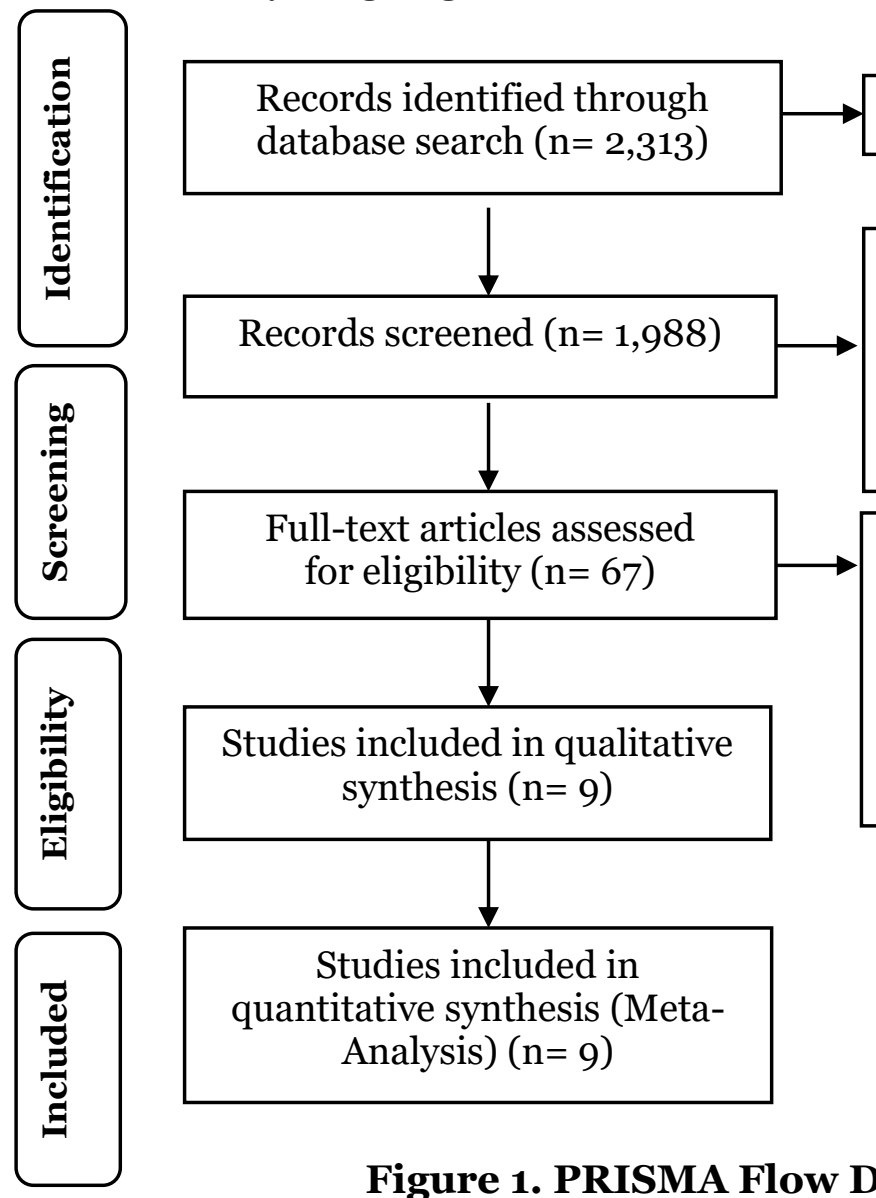

Duplications removed $(\mathrm{n}=325)$

Records excluded $(n=1,921)$

Not relevant title $=1,856$

Not open access $=27$

Articles not in English $=6$

Not full-text articles $=32$

Full-text articles excluded with

reasons $(n=58)$

Not adjusted $\mathrm{OR}=15$

Effect measure other than aOR $(\mathrm{aRR} / \mathrm{aHR})=13$

Not relevant outcome $=30$

Studies included in qualitative synthesis $(n=9)$

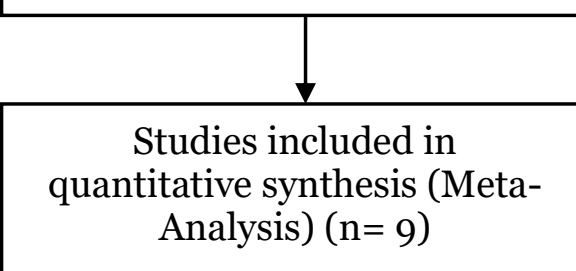

\section{Figure 1. PRISMA Flow Diagram}

\section{RESULTS}

A total of 2,313 articles were identified through electronic databases. After removed the duplications, 1,988 articles were 
screened. Of these, 67 articles were assessed for eligibility criteria. A total of 9 articles that met the quality assessment were included in the quantitative synthesis using Meta-Analysis. Six of the nine included studies were conducted in the United States, two in Europe, including Italy and France, and one in Georgia. The process of selecting articles was presented in Figure 1. The studies quality assessment using the Cohort Study Checklist published by the CASP (Critical Appraisal Skills Program) was presented in Table 2.

Four forest plots were drawn to investigate the pooled effect from each BMI category on the mortality risk of COVID-19 patients. Only class III obesity was evaluated across all nine included studies, while the other categories of high BMI were evaluated across seven studies. The forest plot in Figure 2 showed that COVID-19 patients with overweight had 1.10 times as many mortality risks as patients with normal $\mathrm{BMI}(\mathrm{aOR}=1.10 ; 95 \% \mathrm{CI}=1.00$ to 1.21), but it was not statistically significant $(\mathrm{p}=0.060)$. The $\mathrm{I}^{2}$ was $48 \%$, indicating low heterogeneity in included studies; thus, a fixed-effect model was used. The funnel plot in Figure 3 showed asymmetrical distribution of plots with five plots on the left and two on the right. It indicated publication bias with the underestimated effect of overweight across studies.

The forest plot in Figure 4 showed that COVID-19 patients with class I obesity had 1.16 times as many mortality risks as patients with normal $\mathrm{BMI}(\mathrm{aOR}=1.16$; 95\% $\mathrm{CI}=0.87$ to 1.55 ), but it was not statistically significant $(\mathrm{p}=0.300)$. The $\mathrm{I}^{2}$ was $71 \%$, indicating moderate heterogeneity in included studies; thus, a random-effect model was used. The funnel plot in Figure 5 showed symmetrical distribution of plots with four plots on the left and three on the right, indicating no publication bias.

The forest plot in Figure 6 showed that COVID-19 patients with class II obesity BMI range had 1.54 times as many mortality risks as normal $\mathrm{BMI}(\mathrm{aOR}=1.54$; $95 \% \mathrm{CI}=1.11$ to 2.13 ), and it was statistically significant $(\mathrm{p}=0.009)$.

The $\mathrm{I}^{2}$ was $65 \%$, indicating moderate heterogeneity in included studies; thus, a random-effect model was used. The funnel plot in Figure 7 showed symmetrical distribution of plots with four plots on the left and three on the right, indicating no publication bias.

The forest plot in Figure 8 showed that COVID-19 patients with class III obesity BMI range had 1.77 times as many mortality risks as normal $\mathrm{BMI}(\mathrm{aOR}=1.77$; 95\% CI $=1.27$ to 2.47 ), and it was statistically significant $(\mathrm{p}=0.001)$. The $\mathrm{I}^{2}$ was $86 \%$, indicating high heterogeneity in included studies; thus, a random-effect model was used. The funnel plot in Figure 9 showed symmetrical distribution of plots with five plots on the left and four on the right, indicating no publication bias. 
Table 2. Critical Appraisal Checklist for Cohort Study

\begin{tabular}{|c|c|c|c|c|c|c|c|c|c|c|}
\hline \multirow[b]{2}{*}{ No. } & \multirow[b]{2}{*}{ Critical Appraisal Checklists } & \multicolumn{7}{|c|}{$\begin{array}{c}\text { Publication } \\
\text { (Authors and Year) } \\
\end{array}$} & \multirow[b]{2}{*}{$\begin{array}{c}\text { Shah } \\
\text { et al. } \\
\text { (2020) }\end{array}$} & \multirow[b]{2}{*}{$\begin{array}{l}\text { Yoshida } \\
\text { et al. } \\
\text { (2021) }\end{array}$} \\
\hline & & $\begin{array}{l}\text { Czernichow } \\
\text { et al. } \\
(\mathbf{2 0 2 0}) \\
\end{array}$ & $\begin{array}{c}\text { Guerson } \\
\text { Gil et al. } \\
(2020) \\
\end{array}$ & $\begin{array}{l}\text { Gupta } \\
\text { et al. } \\
(2020)\end{array}$ & $\begin{array}{l}\text { Halasz } \\
\text { et al. } \\
(\mathbf{2 0 2 0}) \\
\end{array}$ & $\begin{array}{c}\text { Kim } \\
\text { et al. } \\
(\mathbf{2 0 2 1})\end{array}$ & $\begin{array}{c}\text { Klang } \\
\text { et al. } \\
(\mathbf{2 0 2 0})\end{array}$ & $\begin{array}{l}\text { Nyabera } \\
\text { et al. } \\
(\mathbf{2 0 2 0}) \\
\end{array}$ & & \\
\hline 1. & $\begin{array}{l}\text { Did the study address a clearly focused } \\
\text { issue? }\end{array}$ & 1 & 1 & 1 & 1 & 1 & 1 & 1 & 1 & 1 \\
\hline 2. & $\begin{array}{l}\text { Was the cohort recruited in an } \\
\text { acceptable way? }\end{array}$ & 1 & 1 & 1 & 1 & 1 & 1 & 1 & 1 & 1 \\
\hline 3. & $\begin{array}{l}\text { Was the exposure COVID-19 accurately } \\
\text { measured to minimize bias? }\end{array}$ & 1 & 1 & 1 & 1 & 1 & 1 & 1 & 1 & 1 \\
\hline 4. & $\begin{array}{l}\text { Was the outcome (status mortality) } \\
\text { accurately measured to minimize bias? }\end{array}$ & 1 & 1 & 1 & 1 & 1 & 1 & 1 & 1 & 1 \\
\hline 5. & $\begin{array}{l}\text { Have the authors identified all- } \\
\text { important confounding factors? Have } \\
\text { the authors took account of the } \\
\text { confounding factors in the design and/ } \\
\text { or analysis? }\end{array}$ & 1 & 1 & 1 & 1 & 1 & 1 & 1 & 1 & 1 \\
\hline 6. & $\begin{array}{l}\text { Was the follow-up of subjects complete } \\
\text { enough? Was the follow-up of subjects } \\
\text { long enough? }\end{array}$ & 1 & 1 & 1 & 1 & 1 & 1 & 1 & 1 & 1 \\
\hline $7 \cdot$ & $\begin{array}{l}\text { Was the result of this study reported in } \\
\text { aOR? }\end{array}$ & 1 & 1 & 1 & 1 & 1 & 1 & 1 & 1 & 1 \\
\hline 8. & How precise were the results? & 1 & 1 & 1 & 1 & 1 & 1 & 1 & 1 & 1 \\
\hline 9. & Do you believe the results? & 1 & 1 & 1 & 1 & 1 & 1 & 1 & 1 & 1 \\
\hline 10. & $\begin{array}{l}\text { Can the results be applied to the local } \\
\text { population? }\end{array}$ & 1 & 1 & 1 & 1 & 1 & 1 & 1 & 1 & 1 \\
\hline 11. & $\begin{array}{l}\text { Do the results of this study fit with } \\
\text { other available evidence? }\end{array}$ & 1 & 1 & 1 & 1 & 1 & 1 & 1 & 1 & 1 \\
\hline \multirow[t]{2}{*}{12.} & $\begin{array}{l}\text { What are the implications of this study } \\
\text { for practice? }\end{array}$ & 1 & 1 & 1 & 1 & 1 & 1 & 1 & 1 & 1 \\
\hline & Total & 12 & 12 & 12 & 12 & 12 & 12 & 12 & 12 & 12 \\
\hline
\end{tabular}

$\mathrm{Yes}=1 ; \mathrm{No}=\mathrm{O}$ 
Table 1. Characteristics of included studies for Meta-Analysis

\begin{tabular}{|c|c|c|c|c|c|c|c|c|}
\hline \multirow{2}{*}{$\begin{array}{l}\text { Author } \\
\text { (Year) }\end{array}$} & \multirow[b]{2}{*}{ Country } & \multirow{2}{*}{$\begin{array}{l}\text { Study } \\
\text { Design }\end{array}$} & \multicolumn{2}{|r|}{ Sample } & \multirow{2}{*}{$\begin{array}{c}\mathbf{P} \\
\text { (Population) }\end{array}$} & \multirow{2}{*}{$\begin{array}{c}\text { I } \\
\text { (Intervention) }\end{array}$} & \multirow{2}{*}{$\begin{array}{c}\mathrm{C} \\
\text { (Comparison) }\end{array}$} & \multirow{2}{*}{$\begin{array}{c}\text { O } \\
\text { (Outcome) }\end{array}$} \\
\hline & & & COVID-19 & $\begin{array}{c}\text { BMI Range } \\
\left(\mathrm{kg} / \mathrm{m}^{2}\right)\end{array}$ & & & & \\
\hline $\begin{array}{l}\text { Halasz et } \\
\text { al. } \\
(2020)\end{array}$ & Italy & $\begin{array}{l}\text { Retro- } \\
\text { spective } \\
\text { Cohort }\end{array}$ & $\begin{array}{l}\text { Total }=242 \\
\text { Non survivor } \\
=78\end{array}$ & $\begin{array}{l}25-29 \cdot 9=104(41 \%), \\
30-34 \cdot 9=11(14 \%), \\
35-39 \cdot 9=3(3 \%), \\
\geq 40=5(6 \%)\end{array}$ & $\begin{array}{l}\text { Hospitalized COVID- } \\
19 \text { patients }\end{array}$ & $\begin{array}{l}\text { COVID-19 } \\
\text { patients with } \\
\text { higher BMI }\end{array}$ & $\begin{array}{l}\text { COVID-19 } \\
\text { patients with } \\
\text { normal BMI }\end{array}$ & Mortality \\
\hline $\begin{array}{l}\text { Gupta et } \\
\text { al. } \\
(2020)\end{array}$ & $\begin{array}{l}\text { United } \\
\text { States }\end{array}$ & $\begin{array}{l}\text { Prospec- } \\
\text { tive Cohort }\end{array}$ & Total $=2,215$ & Not stated & $\begin{array}{l}\text { Hospitalized COVID- } \\
19 \text { patients }\end{array}$ & $\begin{array}{l}\text { COVID-19 } \\
\text { patients with } \\
\text { higher BMI }\end{array}$ & $\begin{array}{l}\text { COVID-19 } \\
\text { patients with } \\
\text { normal BMI }\end{array}$ & Mortality \\
\hline $\begin{array}{l}\text { Czernich } \\
\text { ow et al. } \\
(2020)\end{array}$ & France & $\begin{array}{l}\text { Prospec- } \\
\text { tive Cohort }\end{array}$ & $\begin{array}{l}\text { Total }=4,056 \\
\text { Non survivor } \\
=483\end{array}$ & $\begin{array}{l}25-29 \cdot 9=239 \\
30-34 \cdot 9=145 \\
35-39 \cdot 9=63 \\
\geq 40=36\end{array}$ & $\begin{array}{l}\text { Hospitalized COVID- } \\
19 \text { patients }\end{array}$ & $\begin{array}{l}\text { COVID-19 } \\
\text { patients with } \\
\text { higher BMI }\end{array}$ & $\begin{array}{l}\text { COVID-19 } \\
\text { patients with } \\
\text { normal BMI }\end{array}$ & Mortality \\
\hline $\begin{array}{l}\text { Shah et } \\
\text { al. } \\
(2020)\end{array}$ & Georgia & $\begin{array}{l}\text { Retro- } \\
\text { spective } \\
\text { Cohort }\end{array}$ & $\begin{array}{l}\text { Total }=522 \\
\text { Non survivor }= \\
92(17.6 \%)\end{array}$ & $\begin{array}{l}\geq 30=52(62 \%) \\
\geq 40=23(25 \%)\end{array}$ & $\begin{array}{l}\text { Hospitalized COVID- } \\
19 \text { patients }\end{array}$ & $\begin{array}{l}\text { COVID-19 } \\
\text { patients with } \\
\text { higher BMI }\end{array}$ & $\begin{array}{l}\text { COVID-19 } \\
\text { patients with } \\
\text { normal BMI }\end{array}$ & Mortality \\
\hline $\begin{array}{l}\text { Guerson- } \\
\text { Gil et al. } \\
(2021)\end{array}$ & New York & $\begin{array}{l}\text { Retro- } \\
\text { spective } \\
\text { Cohort }\end{array}$ & Total $=3,530$ & $\begin{array}{l}25-29 \cdot 9=700 \\
30-34 \cdot 9=809 \\
\geq 35-39 \cdot 9=663\end{array}$ & $\begin{array}{l}\text { Hospitalized COVID- } \\
19 \text { patients }\end{array}$ & $\begin{array}{l}\text { COVID-19 } \\
\text { patients with } \\
\text { higher BMI }\end{array}$ & $\begin{array}{l}\text { COVID-19 } \\
\text { patients with } \\
\text { normal BMI }\end{array}$ & Mortality \\
\hline $\begin{array}{l}\text { Yoshida } \\
\text { et al. } \\
(2021)\end{array}$ & USA & $\begin{array}{l}\text { Retro- } \\
\text { spective } \\
\text { Cohort }\end{array}$ & Total $=776$ & $\begin{array}{l}25-29.9=198(25.7 \%), \\
30-34 \cdot 9=157(20.4 \%), \\
35-39.9=118(15 \cdot 3 \%), \\
\geq 40=134(17.4 \%)\end{array}$ & $\begin{array}{l}\text { Hospitalized COVID- } \\
19 \text { patients }\end{array}$ & $\begin{array}{l}\text { COVID-19 } \\
\text { patients with } \\
\text { higher BMI }\end{array}$ & $\begin{array}{l}\text { COVID-19 } \\
\text { patients with } \\
\text { normal BMI }\end{array}$ & Mortality \\
\hline $\begin{array}{l}\text { Klang et } \\
\text { al. } \\
(2020)\end{array}$ & New York & $\begin{array}{l}\text { Retro- } \\
\text { spective } \\
\text { Cohort }\end{array}$ & $\begin{array}{l}\text { Total }=572 \\
\text { Non survivor }= \\
60(10.5 \%)\end{array}$ & $\begin{array}{l}\geq 30=16(26.7 \%) \\
\geq 40=19(31.7 \%)\end{array}$ & $\begin{array}{l}\text { Adult patients aged } \\
<50 \text { years diagnosed } \\
\text { with COVID-19 in } \\
\text { hospital }\end{array}$ & $\begin{array}{l}\text { COVID-19 } \\
\text { patients with } \\
\text { higher BMI }\end{array}$ & $\begin{array}{l}\text { COVID-19 } \\
\text { patients with } \\
\text { normal BMI }\end{array}$ & Mortality \\
\hline $\begin{array}{l}\text { Nyabera } \\
\text { et al. } \\
(2020)\end{array}$ & New York & $\begin{array}{l}\text { Retro- } \\
\text { spective } \\
\text { Cohort }\end{array}$ & Total $=290$ & $\begin{array}{l}25-29.9=75(25.9 \%), \\
30-34 \cdot 9=52(17.9 \%), \\
35-39.9=17(5.9 \%), \\
\geq 40=20(6.9 \%)\end{array}$ & $\begin{array}{l}\text { Hospitalized COVID- } \\
19 \text { patients }\end{array}$ & $\begin{array}{l}\text { COVID-19 } \\
\text { patients with } \\
\text { higher BMI }\end{array}$ & $\begin{array}{l}\text { COVID-19 } \\
\text { patients with } \\
\text { normal BMI }\end{array}$ & Mortality \\
\hline $\begin{array}{l}\text { Kim et al. } \\
(2021)\end{array}$ & New York & $\begin{array}{l}\text { Retro- } \\
\text { spective } \\
\text { Cohort }\end{array}$ & Total $=10,861$ & $\begin{array}{l}25-29 \cdot 9=4,021 \\
30-34 \cdot 9=2,345 \\
35-39 \cdot 9=990 \\
\geq 40=755\end{array}$ & $\begin{array}{l}\text { Hospitalized COVID- } \\
19 \text { patients }\end{array}$ & $\begin{array}{l}\text { COVID-19 } \\
\text { patients with } \\
\text { higher BMI }\end{array}$ & $\begin{array}{l}\text { COVID-19 } \\
\text { patients with } \\
\text { normal BMI }\end{array}$ & Mortality \\
\hline
\end{tabular}


Maung et al./ High Body Mass Index and Mortality Risk in Patients with COVID-19

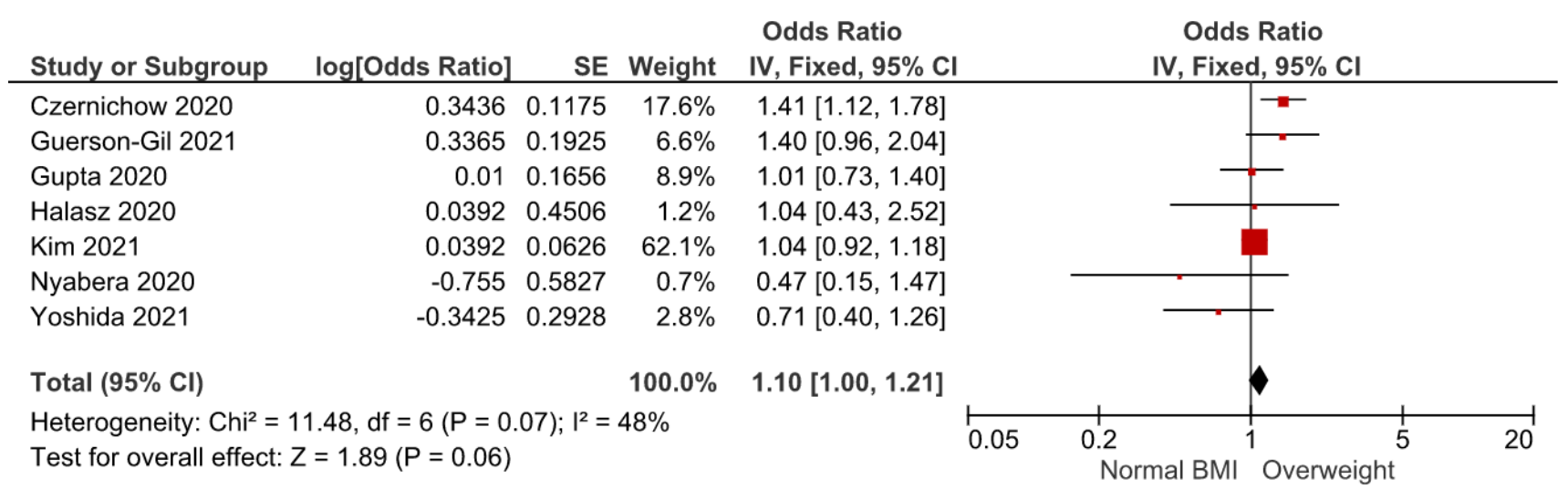

\section{Figure 2. Forest Plot of Association between Overweight and Mortality Risk in Hospitalized COVID-19 Patients}

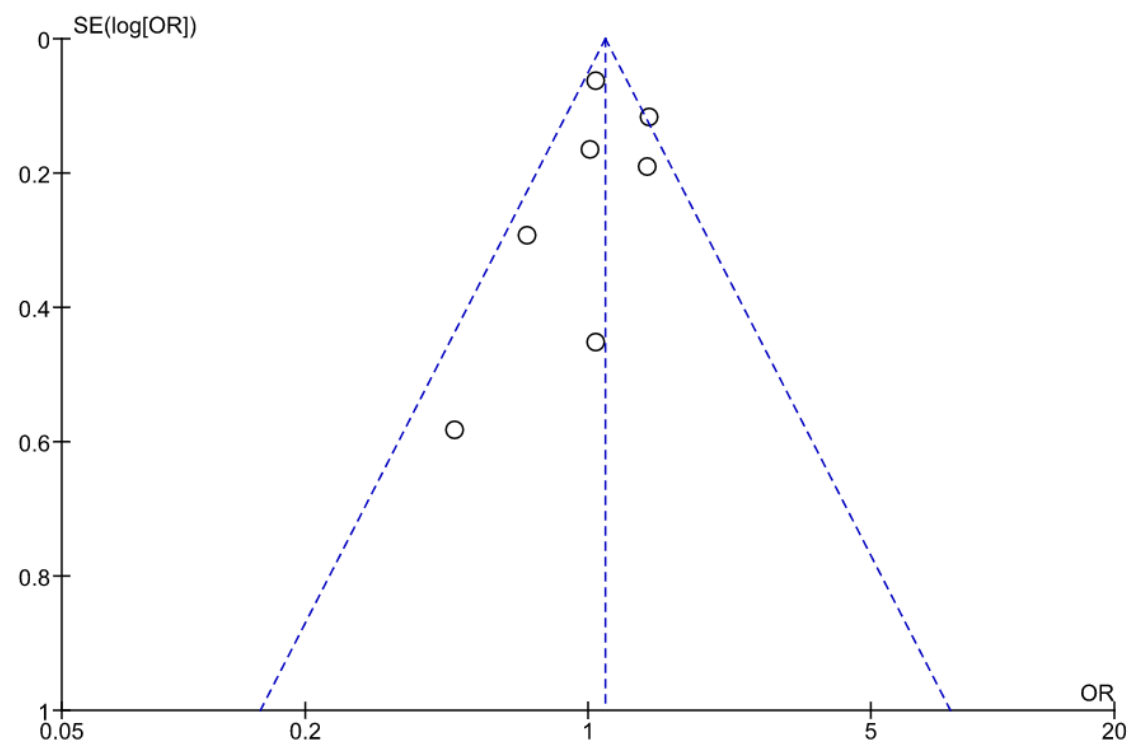

\section{Figure 3. Funnel Plot of Association between Overweight and Mortality Risk in Hospitalized COVID-19 Patients}

\begin{tabular}{|c|c|c|c|c|c|c|c|c|c|}
\hline Study or Subgroup & log[Odds Ratio] & SE & Weight & $\begin{array}{c}\text { Odds Ratio } \\
\text { IV, Random, } 95 \% \mathrm{CI}\end{array}$ & & & $\begin{array}{r}\text { Odds } \\
\text { IV, Rando }\end{array}$ & $\begin{array}{l}\text { s Ratio } \\
\text { om, } 95 \% \mathrm{Cl}\end{array}$ & \\
\hline Czernichow 2020 & 0.6366 & 0.1352 & $21.0 \%$ & $1.89[1.45,2.46]$ & & & & $\rightarrow$ & \\
\hline Guerson-Gil 2021 & 0.3075 & 0.2644 & $13.9 \%$ & $1.36[0.81,2.28]$ & & & & & \\
\hline Gupta 2020 & -0.0305 & 0.1738 & $18.8 \%$ & $0.97[0.69,1.36]$ & & & & & \\
\hline Halasz 2020 & 0.4121 & 0.5538 & $5.4 \%$ & $1.51[0.51,4.47]$ & & & & & \\
\hline Kim 2021 & 0 & 0.0711 & $24.2 \%$ & $1.00[0.87,1.15]$ & & & & & \\
\hline Nyabera 2020 & -0.462 & 0.5854 & $5.0 \%$ & $0.63[0.20,1.98]$ & & & & & \\
\hline Yoshida 2021 & -0.1625 & 0.3133 & $11.7 \%$ & $0.85[0.46,1.57]$ & & & & & \\
\hline Total $(95 \% \mathrm{Cl})$ & & & $100.0 \%$ & $1.16[0.87,1.55]$ & & & & & \\
\hline \multicolumn{5}{|c|}{$\begin{array}{l}\text { Heterogeneity: } \mathrm{Tau}^{2}=0.08 ; \mathrm{Chi}^{2}=20.77, \mathrm{df}=6 \\
\text { Test for overall effect: } Z=1.04(P=0.30)\end{array}$} & 0.05 & 0.2 & rmal BMI & 1 Class I Ob & $\begin{array}{l}5 \\
5 \\
\text { pesity }\end{array}$ \\
\hline
\end{tabular}

Figure 4. Forest Plot of Association between Class I Obesity and Mortality Risk in Hospitalized COVID-19 Patients 
Maung et al./ High Body Mass Index and Mortality Risk in Patients with COVID-19

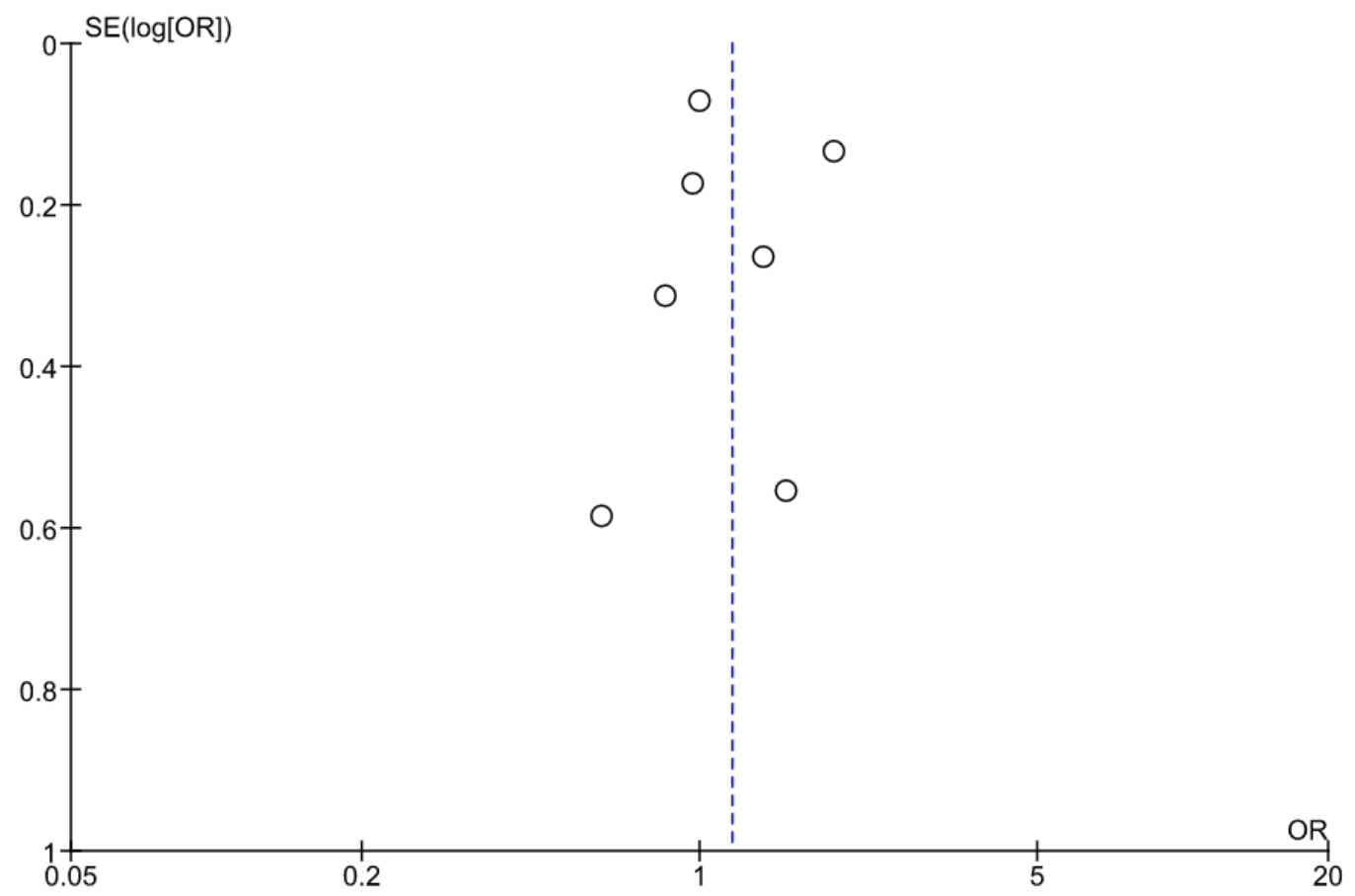

Figure 5. Funnel Plot of Association between Class I Obesity
and Mortality Risk in Hospitalized COVID-19 Patients

Odds Ratio

Odds Ratio

Study or Subgroup log[Odds Ratio] SE Weight IV, Random, $95 \% \mathrm{CI}$

IV, Random, $95 \% \mathrm{Cl}$

$\begin{array}{lrrrr}\text { Czernichow 2020 } & 1.026 & 0.1828 & 20.6 \% & 2.79[1.95,3.99] \\ \text { Guerson-Gil 2021 } & 0.5933 & 0.2778 & 15.5 \% & 1.81[1.05,3.12] \\ \text { Gupta 2020 } & 0.2151 & 0.2173 & 18.7 \% & 1.24[0.81,1.90] \\ \text { Halasz 2020 } & 0.5539 & 0.8802 & 3.1 \% & 1.74[0.31,9.77] \\ \text { Kim 2021 } & 0.2231 & 0.0988 & 25.0 \% & 1.25[1.03,1.52] \\ \text { Nyabera 2020 } & 0 & 0.7073 & 4.6 \% & 1.00[0.25,4.00] \\ \text { Yoshida 2021 } & 0.131 & 0.3448 & 12.5 \% & 1.14[0.58,2.24] \\ & & & \\ \text { Total (95\% Cl) } & & 100.0 \% & 1.54[1.11,2.13] \\ \text { Heterogeneity: } \text { Tau }^{2}=0.10 ; \text { Chi }^{2}=17.03, \mathrm{df}=6(P=0.009) ; \mathrm{I}^{2}=65 \%\end{array}$

y. Tau $=0.10 ; C h 1^{2}=17.03, \mathrm{df}=6(P=0.009), l^{2}=65 \%$

Test for overall effect: $Z=2.61(P=0.009)$

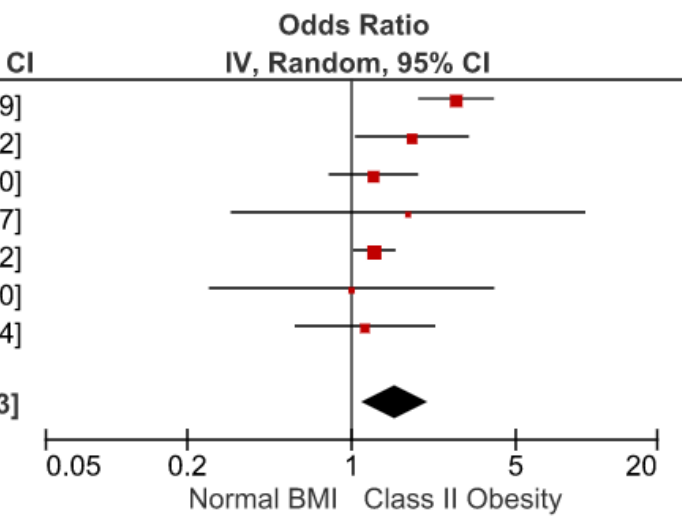

Figure 6. Forest Plot of Association between Class II Obesity and Mortality Risk in Hospitalized COVID-19 Patients 
Maung et al./ High Body Mass Index and Mortality Risk in Patients with COVID-19

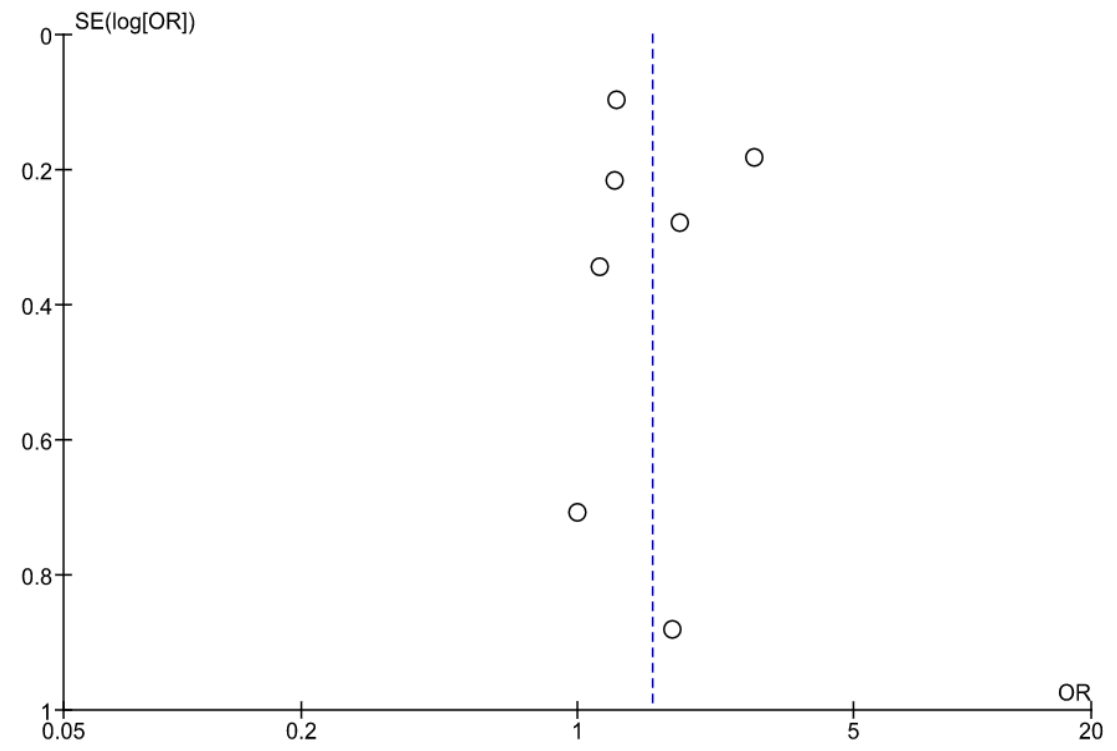

Figure 7. Funnel Plot of Association between Class II Obesity and Mortality Risk in Hospitalized COVID-19 Patients

\begin{tabular}{|c|c|c|c|c|c|c|c|c|c|}
\hline Study or Subgroup & log[Odds Ratio] & SE & Weight & $\begin{array}{c}\text { Odds Ratio } \\
\text { IV, Random, } 95 \% \mathrm{CI}\end{array}$ & & $\begin{array}{r}\text { Odds } \\
\text { IV, Rand }\end{array}$ & $\begin{array}{l}\text { s Ratio } \\
\text { om, } 95 \% \mathrm{Cl}\end{array}$ & & \\
\hline Czernichow 2020 & 0.9361 & 0.2315 & $13.5 \%$ & $2.55[1.62,4.01]$ & & & & & \\
\hline Guerson-Gil 2021 & 0.0583 & 0.0146 & $18.0 \%$ & $1.06[1.03,1.09]$ & & & F & & \\
\hline Gupta 2020 & 0.4121 & 0.2052 & $14.3 \%$ & $1.51[1.01,2.26]$ & & & & & \\
\hline Halasz 2020 & 1.3635 & 0.7908 & $3.7 \%$ & $3.91[0.83,18.42]$ & & & & & \\
\hline Kim 2021 & 0.4762 & 0.1091 & $16.8 \%$ & $1.61[1.30,1.99]$ & & & $\rightarrow$ & & \\
\hline Klang 2020 & 1.6292 & 0.4063 & $8.9 \%$ & $5.10[2.30,11.31]$ & & & & & \\
\hline Nyabera 2020 & -0.6931 & 0.6873 & $4.6 \%$ & $0.50[0.13,1.92]$ & & & & & \\
\hline Shah 2020 & 0.8286 & 0.3695 & $9.7 \%$ & $2.29[1.11,4.72]$ & & & & & \\
\hline Yoshida 2021 & 0.4947 & 0.3353 & $10.6 \%$ & $1.64[0.85,3.16]$ & & & & & \\
\hline Total $(95 \% \mathrm{Cl})$ & & & $100.0 \%$ & $1.77[1.27,2.47]$ & & & & & \\
\hline \multicolumn{5}{|c|}{$\begin{array}{l}\text { Heterogeneity: } \mathrm{Tau}^{2}=0.16 ; \mathrm{Chi}^{2}=55.63, \mathrm{df}=\varepsilon \\
\text { Test for overall effect: } Z=3.37(P=0.0008)\end{array}$} & 0.05 & 0.2 & 1 Class III Ob & $\begin{array}{l}1 \\
\text { besity }\end{array}$ & 20 \\
\hline
\end{tabular}

Figure 8. Forest Plot of Association between Class III Obesity
and Mortality Risk in Hospitalized COVID-19 Patients 


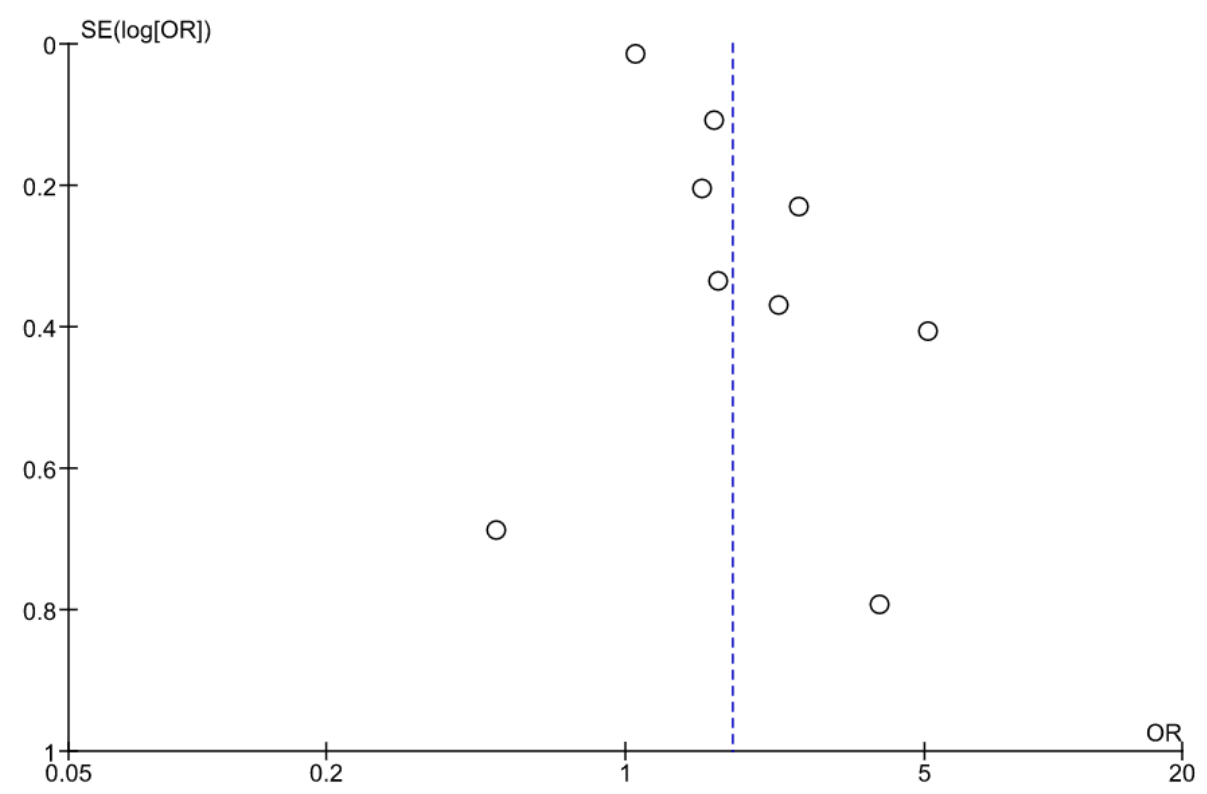

\section{Figure 9. Funnel Plot of Association between Class III Obesity and Mortality Risk in Hospitalized COVID-19 Patients}

\section{DISCUSSION}

This systematic review and Meta-Analysis investigated the association of high BMI and the risk of mortality in hospitalized COVID-19 patients based on four categories of high BMI range. All nine studies included in this review used an observational cohort design only to strengthen the validity of the results.

The findings of this study elucidated that a high BMI was associated with an increased risk of mortality in COVID-19 patients. Furthermore, this study discovered a possible dose-response relationship effect of higher BMI on mortality risk. There was a higher mortality risk of COVID-19 in class III obesity patients $(\mathrm{aOR}=1.77 ; 95 \% \mathrm{CI}=1.27$ to $2.47 ; \mathrm{p}=$ o.001) compared to overweight $(\mathrm{aOR}=1.10$; $95 \% \mathrm{CI}=1.00$ to $1.21 ; \mathrm{p}=0.060$ ), class $\mathrm{I}$ $(\mathrm{aOR}=1.16 ; 95 \% \mathrm{CI}=0.87$ to $1.55 ; \mathrm{p}=$ $0.300)$, and class II obesity $(\mathrm{aOR}=1.54$; 95\% $\mathrm{CI}=1.11$ to 2.13 ; $\mathrm{p}=0.009$ ).

The patients with BMI ranges of class II and III obesity demonstrated a moderate causal relationship, and it was statistically significant. In contrast, overweight and class I obesity demonstrated a weak causal relationship on the risk of COVID-19 mortality, and it was not statistically significant.

This study results aligned with the study conducted by Palaiodimos et al. (2020) with significant results of severe obesity $\left(\mathrm{BMI}=\geq 35 \mathrm{~kg} / \mathrm{m}^{2}\right)$ increased the risk of in-hospital mortality $(\mathrm{aOR}=3.78$; 95\% CI= 1.45 to 9.83) along with the need of oxygen requirements and intubation. A large cohort study from 88 US hospitals also revealed that obesity classes I, II, and III were associated with progressively higher risks of in-hospital death or mechanical ventilation compared to individuals of normal weight (Hendren et al., 2021). A J-shape association between BMI and risk of death was occurred, even after adjusting for obesity-related comorbidities, including metabolic and cardiovascular diseases (Tartof et al., 2020).

Besides COVID-19 pathogenesis, several mechanisms increase disease severity in obese patients. COVID-19 patients with obesity had significantly higher acute-phase reactants such as serum ferritin, erythroncyte sedimentation rate, C-reactive protein 
(CRP), and D-dimer (Mehanna et al., 2021). They also have a higher rate of proinflammatory cytokine transcription, including tumor necrosis factor- $\alpha$ (TNF- $\alpha$ ), interleukin-1 (IL-1), and interleukin-6 (IL-6). These inflammatory cytokines then upregulate the concentration and synthesis of CRP in the liver and develop feedback loops of acute phase reactants and chronic inflammation (Guglielmi et al., 2021; Chiappetta et al., 2020).

In addition, adipose tissue also expressed angiotensin II (ATII), which was recognized as a receptor for SARS-CoV-2 antigen, resulting in a more vulnerable COVID-19 infection target (Seidu et al., 2021). The risk of thromboembolism, reduced lung mechanism, and proinflammatory cytokine cascade due to excess fat accumulation may add to the severity of COVID-19 infection (Mafort et al., 2016; Mehanna et al., 2021).

There are limitations to this review. The majority of included studies were from America (mostly from the USA), which may restrict the generalized ability to nonAmerica individuals, including Asia, the Mediterranean, and the Middle East. Due to data constraints, only class III obesity can be applied to all included nine studies. Lastly, the researcher only included articles written in English, which may have resulted in non-English articles omission.

In conclusion, severe obesity may be a strong risk factor for mortality in COVID-19 patients with the potential of dose-response relationship effect of higher BMI on mortality risk. Future studies should consider subgroup analysis accounting clinical and demographic characteristics and doseresponse relationship Meta-Analysis to ensure the evidence of the relationship between high BMI and mortality risk of COVID-19 patients. Also, the policymakers should consider the COVID-19 vaccination priority for the obese population, especially with high-risk morbid obesity.

\section{AUTHOR CONTRIBUTION}

Study conception and design: Yuzana Maung, Bhisma Murti

Data collection: Yuzana Maung, Eti Poncorini Pamungkasari

Data analysis and interpretation: Yuzana Maung, Bhisma Murti

Drafting of the article: All authors

Critical revision of the article: Eti Poncorini Pamungkasari, Bhisma Murti

\section{CONFLICT OF INTEREST}

The authors declare there were no conflicts of interest.

\section{FUNDING AND SPONSORSHIP}

Self-funded.

\section{ACKNOWLEDGEMENT}

We would like to thank the Masters Program in Public Health, Universitas Sebelas Maret, for supporting this manuscript's publication. The contents of this manuscript were entirely the responsibility of the authors.

\section{REFERENCES}

Chen N, Zhou M, Dong X, Qu J, Gong F, Han Y, Qiu Y et al. (2020). Epidemiological and clinical characteristics of 99 cases of 2019 novel coronavirus pneumonia in Wuhan, China: a descriptive study. Lancet. 395(10223): 507-513. DOI: 10.1016/So140-6736(20)30211-7.

Chiappetta S, Sharma A, Bottino V, Stier C (2020). COVID-19 and the role of chronic inflammation in patients with obesity. Int J Obes. 44: 1790-1792. DOI: $10.1038 / \mathrm{s} 41366-020-0597-4$

Docherty AB, Harrison EM, Green CA, Hardwick HE, Pius R, Norman L, 
Holden KA, et al. (2020). Features of 20,133 UK patients in hospital with covid-19 using the ISARIC WHO Clinical Characterisation Protocol: Prospective observational cohort study. BMJ. 369:1985. DOI: 10.-1136/bmj.m1985.

Giacomelli A, Ridolfo AL, Milazzo L, Oreni L, Bernacchia D, Siano M, Bo-nazzetti C, et al. (2020). 30-day mortality in patients hospitalized with COVID-19 during the first wave of the Italian epidemic: A prospective cohort study. Pharmacol Res. 158:104931. DOI: 10.1016/j.phrs.2020ᄀ.104ᄀ9-31.

Guglielmi V, Colangeli L, Adamo MD, Sbraccia P (2021). Susceptibility and Severity of Viral Infections in Obesity: Lessons from Influenza to COVID-19. Does Leptin Play a Role? Int. J. Mol. Sci, 22: 3183. DOI.org/ᄀ10.3390/ijms22063183

Hajifathalian K, Kumar S, Newberry C, Shah S, Fortune B, Krisko T, Pujols SO, et al. (2020). Obesity is Associated with Worse Outcomes in COVID-19: Analysis of Early Data from New York City. Obesity. 28(9): 1606-1612. doi: 10.1002/oby.22923.

Hendren N, Lemos J, Ayers C, Das S, Rao A, Carter S, Rosenblatt A, et al. (2021). Association of Body Mass Index and Age With Morbidity and Mortality in Patients Hospitalized With COVID-19. Circulation. 143: 135-144. DOI: 10.1161/CIRCULATIONAHA.120.051936

Jain S, Chaves S (2011). Obesity and Influenza. Clin. Infect. Dis. 53(5): 422-424. DOI: $10.1093 / \mathrm{cid} / \mathrm{cir} 448 \mathrm{f}$

Mafort TT,Rufino R, Costa CH, Lopes AJ (2016). Obesity: Systemic and pulmonary complications, biochemical abnormalities, and impairment of lung function. Multidiscip Respir Med. 11(28): 1-11. DOI: 10.1186/s40248- 016-0066-z.

Mehanna O, El Askary A, El Esawy B, FathAlla T, Gharib AF (2021). Impact of Obesity and Its Associated Comorbid Conditions on COVID-19 Presentation. Diabetes Metab Syndr Obes. 14: 409-415. DOI: org/10.2147/-DMSO.S287779

Mehta P, McAuley DF, Brown M, Sanchez E, Tattersall RS, Manson JJ (2020). COVID-19: consider cytokine storm syndromes and immunosuppression. The Lancet. 395(10229): 1033-1034. DOI: $10.1016 /$ So140-6736(20)30628o.

Palaidimos L, Kokkinidis D, Li W, Karamanis D, Ognibene J, Arora S, Southern W, et al. (2020). Severe obesity, increasing age and male sex are independently associated with worse in-hospital outcomes, and higher in-hospital mortality, in a cohort of patients with COVID-19 in the Bronx, New York. Metab.: Clin. Exp.108: 154262. DOI: org/10.1016/j.metabol.2020.154262

Petrilli CM, Jones SA, Yang J, Rajagopalan H, Donnell LO,Chernyak Y, Tobin KA et al. (2020). Factors associated with hospital admission and critical illness among 5,279 people with coronavirus disease 2019 in New York City: Prospective cohort study. BMJ. 369. DOI: 10.1136/bmj.m1966.

Seidu S, Gillies C, Zaccardi F, Kunutsor S, Boyce J, Yates T, Singh A, et al. (2020). The impact of obesity on severe disease and mortality in people with SARS-CoV-2: A systematic review and meta-analysis. Endocrinol Diabetes Metab. 4(1): eoo176. DOI: 10.1002/edm2.176

Tartof S, Qian L, Hong V, Wei R, Nadjafi R, Fischer H, Li Z, et al. (2020). Obesity and mortality among patients diag- 
Maung et al./ High Body Mass Index and Mortality Risk in Patients with COVID-19

nosed with COVID-19: Results from an Integrated Health Care Organization. Ann Intern Med. DOI:10.7326/M20-3742.

Tsai PH, Lai W, Lin Y, Luo Y, Lin Y, Chen H, Chen $Y$ et al. (2021). Clinical manifestation and disease progression in COVID-19 infection. J Chin Med Assoc. 84(1): 3-8. DOI: 10.1097/JCMA.0000000000000463.

WHO (2020). COVID-19 Weekly Epidemiological Update 22. World Health Organization. 1-3. Retrieved from https://www.who.int/docs/default- source/coronaviruse/situation-reports/weekly_epidemio-logical_update_-22.-pdf.

WHO (2021). WHO Coronavirus (COVID19) Dashboard. World Health Organization. Retrieved from https://-covid19.who.int/.

Wilding J, Ralston J (2021). COVID-19 and Obesity: The 2021 Atlas. World-obesityday. 1-227. Landon: World Obesity Federation. 